\section{Imaging islets}

\section{By Lauren Martz, Staff Writer}

Harvard Medical School researchers have noninvasively imaged pancreatic inflammation, one of the earliest signs of type 1 diabetes, and were able to distinguish between healthy controls and diabetes patients. ${ }^{1}$ The tool could be used to provide an early diagnosis of the disease and to rapidly gauge the efficacy of therapeutics in development.

Type 1 diabetes involves an autoimmune assault on insulin-producing $\beta$ cells in the pancreas. The initial immune inflammation in the pancreas, called insulitis, begins years before the emergence of overt disease and is both an early sign and pathological driver of disease.

In that early stage, immune cells infiltrate the organ and gradually decrease $\beta$ cell mass until insulin production cannot control blood glucose.

There are no safe and reliable ways to detect insulitis.

Pancreatic biopsy is able to identify insulitis and other features of pancreatic dysfunction, but the method is highly invasive. ${ }^{2}$

Alternatively, measurements of circulating autoantibodies or autoreactive T cells can indicate the early autoimmune pathology $\mathrm{y}^{3}$ but often have sampling errors. In addition, it is unclear whether blood measurements are reflective of events in the pancreas.

"One of the challenges in patients with type 1 diabetes is that we don't have a great way to analyze the immune cells doing the destruction," said Mark Anderson, associate professor in the
"This is one of the few-if not the only-studies able to discriminate between type 1 diabetes patients and those who don't have the disease with noninvasive imaging."

- Mark Anderson, University of California,

San Francisco signal when taken up by macrophages. The signal in the pancreas reflected the amount of nanoparticles that leaked from pancreatic blood vessels into the surrounding pancreatic tissue, where the particles were taken up by macrophages.

Because disruption of the pancreatic vasculature-specifically vessel leakage - is associated with insulitis, the signal correlated with the degree of islet inflammation.

The team tested the strategy in 10 type 1 diabetes patients diagnosed within 6 months and in 12 nondiabetic controls. Patients were imaged a minimum of 3 times: before nanoparticle infusion, immediately after infusion and 48 hours postinfusion.

Images showed that pancreatic volume, controlled for body size, was about $31 \%$ smaller in diabetes patients than in controls, indicating that pancreatic atrophy occurs early in the course of disease.

In addition, there was significantly more nanoparticle accumulation in the pancreas of diabetes patients compared with in the pancreas of controls. That finding suggests vessels in the diseased pancreases were akier and insulitis was present.

"This is one of the few-if not the only-studies able to discriminate between type 1 diabetes patients and those who don't have the disease with noninvasive imaging," Anderson told SciBX.

"The potential for strategies that image insulitis, like the MRI proposed by the authors, is that we may be able to diagnose the injury that leads to type 1 diabetes before the injury is severe enough to actually result in clinical type 1 diabetes," said Melena Bellin, assistant professor of pediatrics and surgery at the University of Minnesota's Division of Pediatric Endocrinology and the Schulze Diabetes Institute. "About $80 \%$ of $\beta$ cells are destroyed before a person with diabetes has deviations in blood sugar."

In addition to earlier diagnosis, Mathis told SciBX that "unlike PET imaging modalities, there is not the issue of radiation exposure, so there should be better application to children and to repeated imaging of the same patient."

"There is potential that this type of imaging could help you determine which type of diabetes you are dealing with because it isn't always clear even with clinical presentation. This could help you determine whether it is type 1 , type 2 or something in between called latent autoimmune diabetes," said Louis Philipson, professor in the Department of Medicine and Department of Pediatrics in the section of endocrinology, diabetes and metabolism, and director of the Kolver Diabetes Center at The University of Chicago.

Philipson is collaborating with Cell Point LLC to study the company's 99mTc-DPTA-Glipizide imaging agent. The agent is in preclinical testing to image functional insulin-secreting cells in type 2 diabetes.

\section{Early warning}

The benefits of better diabetes diagnosis offered by the Harvard imaging technique are unlikely to alter decisions about standard care 
because the only drugs on the market for type 1 disease are insulin and adjunctive treatment with Symlin pramlintide from Amylin Pharmaceuticals Inc.

However, the technique could be used with the handful of treatments in the clinic aimed at modulating type 1 diabetes as early as possible.

"One big area of research in the field of type 1 diabetes is looking at whether we can give therapeutic agents to prevent diabetes in patients who are at risk," said Bellin. "In these studies, an imaging tool that can reliably detect inflammation in the islets might be a useful endpoint for monitoring disease response."

"There are a number of clinical trials to test how therapeutics can halt autoimmune progress, but there aren't good tools to determine how efficacious the interventions are other than indirect measures," said Anderson. "The current tools don't measure what is going on in the pancreas in terms of damage and inflammation."

Alberto Pugliese, professor of medicine, immunology and microbiology and head of the Immunogenetics Program at the University of Miami Miller School of Medicine Diabetes Research Institute, thinks a good strategy "would be to perform the NMR test at diabetes onsetbefore a patient is enrolled in a clinical trial that should block or inhibit islet autoimmunity. A change in the NMR readout after treatment may be correlated with clinical and therapeutic outcomes."

Philipson agreed: "There are several drugs that seek to interfere with the autoimmunity process, and it would be nice to have more insight into that process."

The most advanced immunomodulatory drugs in the clinic for type 1 diabetes include three anti-CD3 antibodies: otelixizumab from Tolerx Inc. and GlaxoSmithKline plc, which is in Phase III testing; teplizumab from MacroGenics Inc. and Eli Lilly and Co., which missed the primary endpoint in a Phase II/III trial in October; and NI-0401 from NovImmune S.A., which is in Phase II trials.

Mathis told SciBX that this imaging technique is already a useful preclinical research tool for both predicting which animals will progress to diabetes and early indication of eventual therapeutic response.

\section{Next steps}

Although the Harvard imaging technique was able to discriminate between newly diagnosed patients and healthy controls, the team now plans to see whether the tool could be used even earlier to identify patients presenting with insulitis who are at risk of progressing to diabetes.

"It is still in early days. We need to see how well it works to predict diabetes and whether it can indeed be used to give an early indication of immunomodulatory drugs," said Mathis.

"In an ideal world, one would want histopathological data to correlate with MRI data and directly validate the method in patients," said Pugliese. "It is a bit of a catch-22 because the lack of easy access to the pancreas is the actual reason why we need this kind of test."

Additionally, he said, "a potential limitation is that sometimes insulitis might present but could be kept in check by regulatory mechanisms, and in this case, I'm not sure that this approach could tell the difference."

Although the results in the paper showed a statistically significant difference between MRI signals for the patient and control groups, Anderson noted that there was some overlap. For example, some individuals in the control group showed stronger signals than some individuals in the patient group.

"The bottom line is that there is a way to go in refining the technology to provide reliable discrimination. Because this team has seen at least some success in distinguishing patients from controls, it is possible that this method could be a more sensitive way to discriminate and a step in the right direction," he said.

Dean Hum, CSO of Genfit S.A., noted that the current lack of precision and specificity of the imaging technique could limit its clinical utility. "While imaging techniques as described in this paper are somewhat noninvasive, it remains that these approaches require access to sophisticated and expensive equipment and some subjective interpretation of results," he said.

Genfit's BMGFT02, a noninvasive and nonimaging biomarker technology to detect $\beta$ cell failure, is in preclinical testing to diagnose diabetes.

Mathis told SciBX that a patent application covering diabetes diagnosis and monitoring using the imaging strategy has been filed in the U.S. and that the IP is available for licensing.

Martz, L. SciBX 3(48); doi:10.1038/scibx.2010.1433

Published online Dec. 16, 2010

\section{REFERENCES}

1. Gaglia, J.L. et al. J. Clin. Invest.; published online Dec. 1, 2010; doi:10.1172/JCl44339

Contact: Diane Mathis, Harvard Medical School, Boston, Mass. e-mail: cbdm@hms.harvard.edu

Contact: Ralph Weissleder, Massachusetts General Hospital, Boston, Mass.

e-mail: rweissleder@mgh.harvard.edu

2. Itoh, N. et al. J. Clin. Invest. 92, 2313-2322 (1993)

3. Lieberman, S.M. \& DiLorenzo, T.P. Tissue Antigens 62, 359-377 (2003)

4. Turvey, S.E. et al. J. Clin. Invest. 115, 2454-2461 (2005)

5. Denis, M.C. et al. Proc. Natl. Acad. Sci. USA 101, 12634-12639 (2004)

COMPANIES AND INSTITUTIONS MENTIONED Amylin Pharmaceuticals Inc. (NASDAQ:AMLN), San Diego, Calif. Cell Point LLC, Centennial, Colo. Eli Lilly and Co. (NYSE:LLY), Indianapolis, Ind. Genfit S.A. (Euronext:ALGFT), Lille, France GlaxoSmithKline plc (LSE:GSK; NYSE:GSK), London, U.K. Harvard Medical School, Boston, Mass.

MacroGenics Inc., Rockville, Md.

Massachusetts General Hospital, Boston, Mass.

Novlmmune S.A., Geneva, Switzerland

Tolerx Inc., Cambridge, Mass.

University of California, San Francisco, Calif.

The University of Chicago, Chicago, III.

University of Miami Miller School of Medicine, Miami, Fla. University of Minnesota, Minneapolis, Minn. 